\title{
Effects of Splenectomy for Osmotic Fragility of Circulating Red Cells in Thoroughbred Horses during Exercise
}

\author{
Kei HANZAWA ${ }^{1 *}$, Katsuyoshi KUBO ${ }^{2},{\text { Makoto } \mathrm{KAI}^{3} \text {, Atsushi HIRAGA }{ }^{3} \text { and Seiki WATANABE }}^{1}$ \\ ${ }^{1}$ Laboratory of Animal Physiology, Tokyo University of Agriculture, 1-1, Sakuragaoka 1 chome, Setagaya-ku \\ Tokyo, 156-8502, ${ }^{2} J a p a n$ Livestock Technology Association, 9-20 Yushima 3 chome, Bunkyo-ku, Tokyo, 113- \\ 0034 and ${ }^{3}$ Equine Research Institute, Japan Racing Association, 321-4, Togami-cho, Utsunomiya, Tochigi, \\ 320-0856, Japan
}

Effects of treadmill exercise on osmotic fragility of circulating erythrocytes in jugular venous blood were investigated before and after splenectomy in 3 control and 3 splenectomized thoroughbreds. Both before and after the operation, the fragility of circulating erythrocytes significantly decreased with warming-up, but significantly increased with incremental exercise, and again significantly decreased with cooling down. The fragility of circulating erythrocytes was lower than that of the autologous splenic red cells, and it gradually decreased after the splenectomy. These result suggest that: 1) frequent accumulation in the spleen accelerates the fragility of erythrocytes; and 2) heavy exercise increases fragility, but light exercise decreases it, regardless of the release of red cells from the spleen into the circulation.

Key words: exercise, erythrocytes, horse, osmotic fragility, splenectomy
J. Equine Sci.

Vol. 10, No. $3 \cdot 4$

pp. $61-65,1999$
Boucher et al. [2] and Sakurai et al. [10] observed that the osmotic fragility of erythrocytes in peripheral blood from racehorses was increased by exercise, but Smith $e t$ al. [12] reported that the osmotic fragility of equine circulating erythrocytes had decreased after exercise. The reason for this discrepancy may be related to the release of red cells from the spleen into the circulation during exercise $[2,10,11]$.

Hanzawa et al. [5-7] suggested that the release of erythrocytes from the spleen into circulation caused by intravenous injection of epinephrine has little effect on the osmotic fragility of circulating erythrocytes from thoroughbreds; heavy exercise increases the osmotic fragility, but light exercise decreases it; and the resting level of circulating erythrocyte fragility was decreased by repeated exercise.

The purpose of this study was to clarify the effects of splenectomy on the osmotic fragility of circulating erythrocytes in horses with exercise. A preliminary report of some of these results has already appeared in the Equine Veterinary Journal: Supplement 18 [5].

This article was submitted October 12, 1999 and was accepted January 21, 2000.

*corresponding author.

\section{Materials and Methods}

Horses: Six thoroughbreds, five 2-year-olds and one 5 -year-old, three males, one gelding and two females, weighing $459 \pm 14 \mathrm{~kg}$, were used for the exercise test. These animals were divided into two groups of three each: control (Group C) vs. splenectomized horses (Group $S)$ [5]. The spleen of Group $S$ was surgically extirpated after removal of the 18 th rib on the left side. In Group $C$ only the 18th rib was removed for a sham operation and the wound immediately sutured [5]. These procedures were conducted at least 6 months before the exercise test.

Exercise training: The horses were conditioned on a treadmill (Mustang-2200, Kagra, Switzerland) 5 days a week for one month before each exercise test as well as during the period between tests. The conditioning regimen consisted of 15 min of treadmill exercise including $2 \mathrm{~min}$ of submaximal exercise.

Exercise test: Before and two months after the operation, each horse performed 2 exercise tests within a 1 week period on a treadmill according to the following protocol: 4 min warming-up at $4 \mathrm{~m} / \mathrm{s}, 5$ min rest (at $0 \%$ incline), 4 min walk at $1.5 \mathrm{~m} / \mathrm{s}, 2 \mathrm{~min}$ trot at $4.4 \mathrm{~m} / \mathrm{s}$, canter to 
gallop at 3 to 4 incremental speeds until the point of fatigue (at 7\% incline) and 20 min cooling down.

Heart rate (HR) was recorded throughout the study with a commercial computer (PE-3000, Polar, Finland) [5].

Blood samples were collected by jugular venepuncture from each horse into heparinised test tubes: $30 \mathrm{~min}$ before the start of the treadmill programme denoted as the resting condition, and immediately following (within 30 sec of) warming-up, incremental exercise test and cooling down. Splenic blood was collected from the extracted spleen as quickly as possible after splenectomy.

Blood lactate $\left[\mathrm{La}^{-}\right]$was measured with an automated lactate analyzer (YSI-1500 Sport, Yellow Springs, USA) [5].

Packed cell volume (PCV) was measured with a microhaematocrit centrifuge.

Osmotic fragility of the erythrocytes after washing with ice-cold Dulbecco's phosphate buffered saline was determined by the haemolysis rate (HL) in a hypotonic salt solution $(0.56 \%)$ with the equipment described earlier [5].

Statistical analysis: Effects of splenectomy were determined by a one way analysis of variance (ANOVA). When the F statistic was significant, multiple comparisons of the means were made by means of $\operatorname{LSD}(\mathrm{P}<0.05)$.

\section{Results}

Effects of splenectomy on osmotic fragility of circulating erythrocytes. The HL of circulating red cells immediately before the operation was similar in Groups $C$ and $S$ (Fig. 1). In contrast, the HL of splenic red cells $(81 \pm 8.9 \%)$ was significantly higher than that of the circulating erythrocytes in Group $S$. No changes were seen in the HL of Group $C$ from the month before the operation to the 8th month after, whereas in Group $S$ there was a significant decrease in the 2nd month after the surgery, and these lower values remained constant until the 8th month. The HL of circulating erythrocytes during the 2nd month after the operation in Group $C$ was significantly higher than in Group $S$ (Fig. 1).

Exercise induced changes in some physiological characteristics in Groups $C$ and $S$ horses. No significant differences were observed between Groups $C$ and $S$ in the running time during the incremental exercise test either before or after the operation. Running velocity during the last incremental exercise test in Group $S$ after splenectomy was significantly lower than in Group $C$ and in both groups before the operation (Table 1).

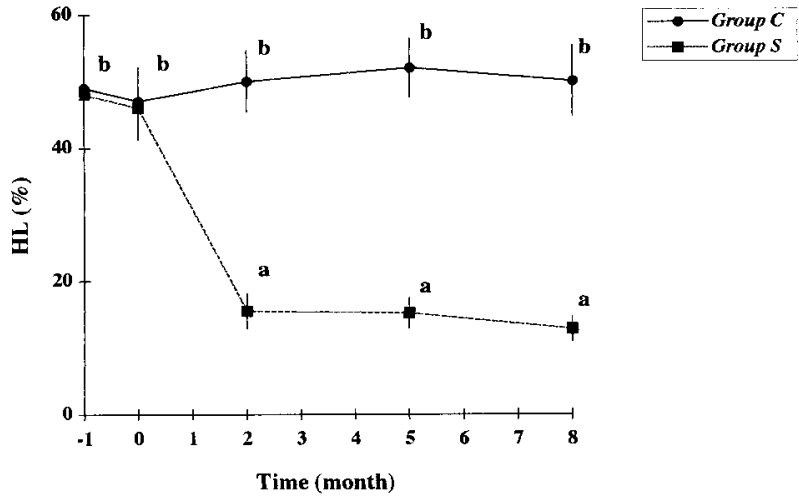

Fig. 1. Effects of splenectomy on osmotic fragility of circulating erythrocytes: haemolysis rate (HL) in a hypotonic salt solution $(0.56 \%)$, in control (Group C) and splenectomized $($ Group $S)$ horses. Differences from each other: $\mathrm{a}<\mathrm{b}$, $\mathrm{P}<0.05$ (LSD).

Table 1. Running time during the incremental exercise test and running velocity during the last incremental exercise test in control (Group C) and splenectomized (Group S) horses before and after operations

\begin{tabular}{lcr}
\hline Operation & Before & \multicolumn{1}{c}{ After } \\
\hline Running time (min) & & \\
Group $C$ & $4.01 \pm 0.33$ & $4.00 \pm 0.22$ \\
Group $S$ & $3.98 \pm 0.26$ & $4.17 \pm 0.21$ \\
Running velocity $(\mathrm{m} / \mathrm{s})$ & & \\
Group $C$ & $10.4 \pm 0.18^{\mathrm{b}}$ & $10.20 \pm 0.19^{\mathrm{b}}$ \\
Group $S$ & $10.4 \pm 0.15^{\mathrm{b}}$ & $8.75 \pm 0.27^{\mathrm{a}}$ \\
\hline
\end{tabular}

Differences from each other: $\mathrm{a}<\mathrm{b}, \mathrm{P}<0.05$ (LSD).

Figure 2 shows the changes in HR during exercise in Groups $C$ and $S$ horses before and after splenectomy. Warming-up significantly increased HR, and incremental exercise increased it much more in all experimental groups, and thereafter cooling down decreased it. No significant differences were observed between Groups $C$ and $S$ in HR before the operation during exercise test. $\mathrm{HR}$ at warming-up and at the last incremental exercise test in Group $S$ after splenectomy was significantly higher than in Group $C$ and in both groups before the operation. There were no differences between Groups $C$ and $S$ in HR after the operation at cooling down.

Figure 3 shows the changes in PCV during exercise in Groups $C$ and $S$ horses before and after splenectomy. Warming-up significantly increased PCV, and incremental exercise increased it much more in all experimental groups except in Group $S$ after splenectomy. In Group $S$ after the operation, there was no change in 


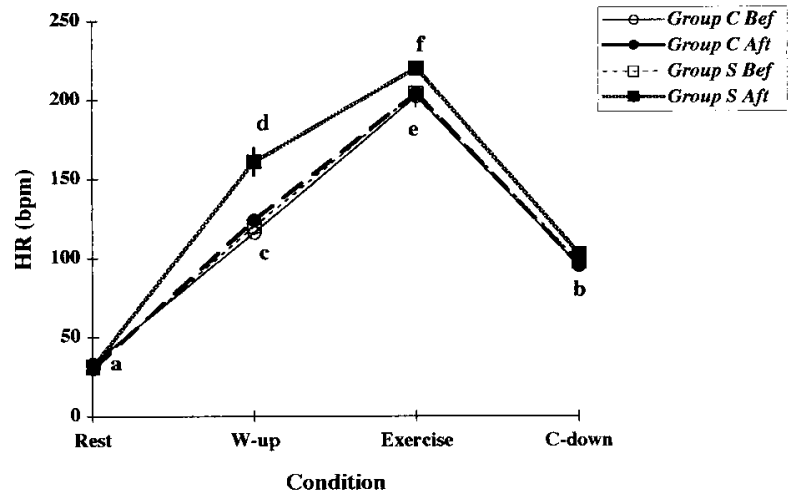

Fig. 2. Changes in heart rate (HR) of control (Group C) and splenectomized (Group $S$ ) horses during exercise before and after splenectomy. Bef: before operation; Aft: after operation; Differences from each other: $\mathrm{a}<\mathrm{b}<\mathrm{c}<\mathrm{d}<\mathrm{e}<\mathrm{f}$, $\mathrm{P}<0.05$ (LSD).

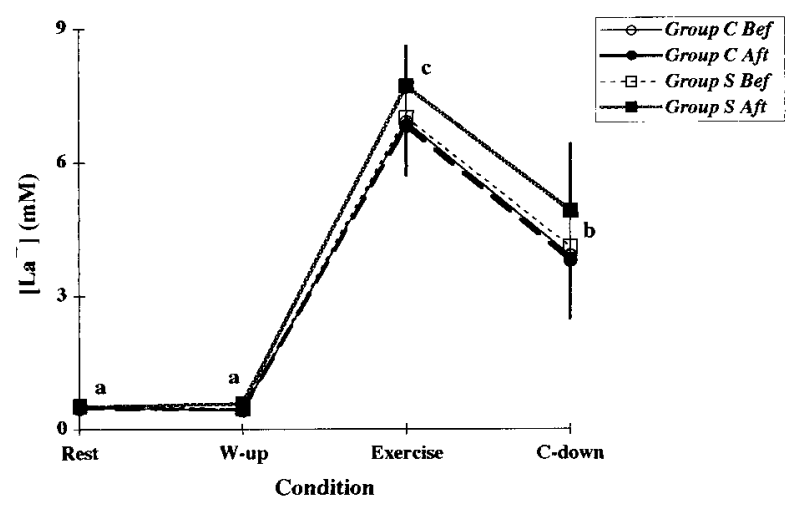

Fig. 4. Changes in lactate concentration of peripheral blood [La ] in control (Group C) and splenectomized (Group S) horses during exercise before and after splenectomy. Bef: before operation; Aft: after operation; Differences from each other: $\mathrm{a}<\mathrm{b}<\mathrm{c}, \mathrm{P}<0.05$ (LSD).

PCV during the exercise test. Cooling down decreased PCV in Group $C$ before and after the operation, and in Group $S$ before the operation to the initial resting level. PCV at warming-up and at the last incremental exercise test in Group $S$ after splenectomy was significantly lower than in Group $C$ and in both groups before the operation.

Figure 4 shows the changes in [ $\left.\mathrm{La}^{-}\right]$during exercise in Groups $C$ and $S$ horses before and after splenectomy. $\left[\mathrm{La}^{-}\right.$ ] did not change with warming-up, but significantly increased with incremental exercise it, and then decreased with cooling down in all experimental groups. There were no differences between Groups $C$ and $S$ horses in $\left[\mathrm{La}^{-}\right]$either before or after the operations.

Figure 5 shows the changes in HL during exercise in

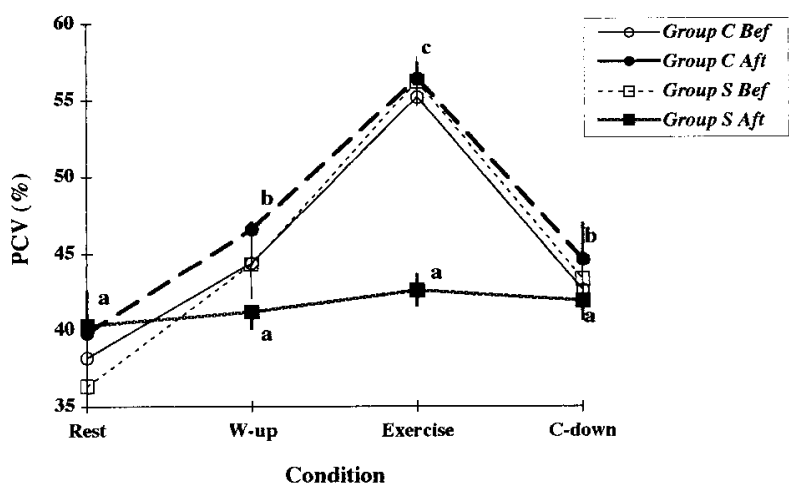

Fig. 3. Changes in packed cell volume (PCV) of peripheral blood in control (Group C) and splenectomized (Group S) horses during exercise before and after splenectomy. Bef: before operation; Aft: after operation; Differences from each other: $\mathrm{a}<\mathrm{b}<\mathrm{c}, \mathrm{P}<0.05$ (LSD).

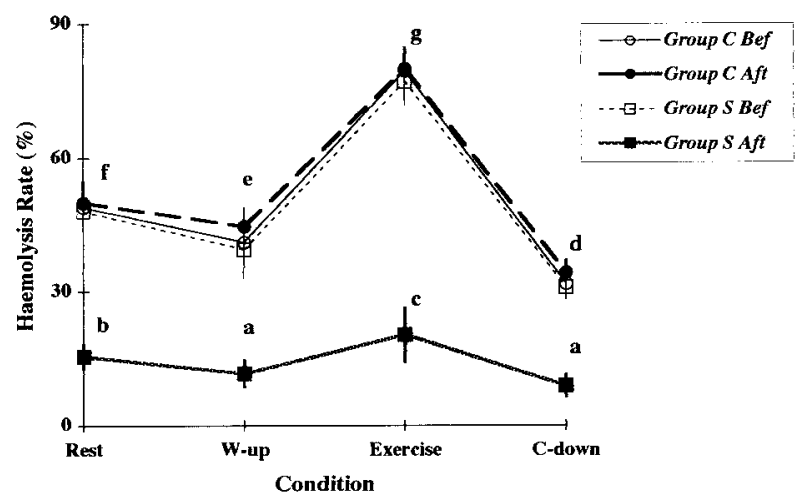

Fig. 5. Changes in osmotic fragility of circulating erythrocytes: haemolysis rate $(\mathrm{HL})$ in a hypotonic salt solution $(0.56 \%)$, in control (Group C) and splenectomized (Group S) horses during exercise before and after splenectomy. Bef: before operation; Aft: after operation; Differences from each other: $\mathrm{a}<\mathrm{b}<\mathrm{c}<\mathrm{d}<\mathrm{e}<\mathrm{f}<\mathrm{g}, \mathrm{P}<0.05$ (LSD).

Groups $C$ and $S$ horses before and after the splenectomy. HL in Group $S$ was significantly lower than in Group $C$ horses regardless of exercise. HL in Group $S$ significantly decreased with warming-up, but significantly increased with incremental exercise, and decreased again with cooling down, similar to controls before splenectomized and/or shame operationed horses.

\section{Discussion}

Running velocity during the last incremental exercise test in Group $S$ after splenectomy was significantly lower 
than in Group C and in both groups before the operation, whereas the $\mathrm{HR}$ at the last incremental exercise test in Groups $S$ after the operation was significantly higher than in the other groups, but there were no differences in running time or $\left[\mathrm{La}^{-}\right]$among in any of experimental groups. Therefore all groups would perform the incremental exercise with similar intensity.

PCV in Group $C$ before and after the operation, and in Group $S$ before splenectomy was significantly increased by exercise, but PCV in Group $S$ after splenectomy was not changed by exercise. This phenomenon suggests that the main cause of the increase in PCV in racehorses during exercise is the release of erythrocytes from the spleen into the circulation [5].

In Group $C$ horses, the HL of circulating erythrocytes was the same before and after sham operation. This suggests that the removal of the 18th rib in both group horses has no effect on the osmotic fragility of erythrocytes [5]. In Group $S$ horses, the HL of erythrocytes in the surgically removed spleen was significantly higher than that of the circulating erythrocytes before and after the operation. The HL of circulating erythrocytes in Group $S$ was significantly decreased by splenectomy. These results suggest that the spleen increases the osmotic fragility of erythrocytes [2], but the fragility of erythrocytes is immediately decreased by the release into the circulation, and the repeated accumulation in the spleen accelerates the fragility of erythrocytes [5].

We also observed that the incremental exercise until the point of fatigue increases the fragility of circulating erythrocytes, and this is supported by Boucher et al. [2], and the warm-up and cooling down decreases the fragility of circulating erythrocytes similarly to that reported by Smith et al. [12], regardless of the release of red cells from the spleen into the circulation [5-7].

The osmotic fragility of circulating erythrocytes after incremental exercise could be accelerated by physical stress: the increase in friction between the red cell membrane and blood vessels caused by a rise in the blood flow rate [3, 4], and chemical stress: the increase in blood lactate caused by metabolic acidosis [4, 7] during exercise in Groups $C$ and $S$ horses. Moreover, in Group Chorses, the fragility of erythrocytes is also promoted by the blood concentration based on the release of erythrocytes from the spleen into the circulation and the release of lysolecithin, which can injure the erythrocytes, from the spleen by the increased secretion of epinephrine $[2,11]$. The reason for an increase in osmotic resistance after cooling down may be a loss of osmotically sensitive erythrocytes due to haemolysis with the incremental exercise. But this hypothesis is unable to account for the decrease in the HL with warming-up.

Ventilatory responses of horses to prolonged submaximal exercise on a treadmill were stimulated by an increase in blood temperature, and then blood $\mathrm{pH}$ was significantly increased by hyperventilation [1]. In a previous report, the increase in $\mathrm{pH}$ and temperature decreased the osmotic fragility of erythrocytes from several mammals and avians in an in vitro condition [9].

Therefore, our results suggest that 1) repeated accumulation in the spleen accelerates the osmotic fragility of erythrocytes; 2) heavy exercise such as cantering and galloping increase physical and chemical stresses, thus accelerating fragility of erythrocytes, but light exercise such as trotting decreases the fragility of erythrocytes, probably due to an increase in blood $\mathrm{pH}$ and temperature, regardless of the release of erythrocytes from the spleen into the circulation; and that 3) release of erythrocytes from the spleen is associated with an increase in the fragility of erythrocytes during heavy exercise [5-7].

\section{Acknowledgment}

This research was partially supported by the Minsitry of Education, Science, Sports and Culture, Grant-in-Aid for Scientific Research (C), 11660287, 1999.

\section{References}

1. Bailey, W., Schott, H., and Slocombe, R. 1995. Ventilatory responses of horses to prolonged submaximal exercise. Equine Vet.J. Suppl. 18: 2328.

2. Boucher, J.H., Lessin, L.S., and McMeekin, R.R. 1985. Echinocytosis: The cause of equine exertional diseases-a hypothesis. pp. 97-112. In: Proceeding of the 5th Annual Scientific Meeting, Association of Equine Sports Medicine. Dynamics of Equine Sports Medicine, Lawrenceville, N.J.

3. Broun, G.O. 1922. Blood destruction during exercise. I. Blood changes occurring in the course of a single day of exercise. J. Exper. Med. 36: 481500.

4. Davidson, R.J.L. 1964. Exersional haemoglobinuria. A report on three cases with studies on the haemolytic mechanism. J. Clin. Pathol. 17: 536-540.

5. Hanzawa, K., Kubo, K., Kai, M., Hiraga, A., and Watanabe, S. 1995. Effects of exercise on 
erythrocytes in normal and splenectomised thoroughbred horses. Equine Vet.J. Suppl. 18: 439442.

6. Hanzawa, K., Kubo, K., Kai, M., Hiraga, A., and Watanabe, S. 1996. Effects of three repetitive loads of incremental exercise on circulating erythrocytes in thoroughbred horses. Pferdeheikunde 12: 502-505.

7. Hanzawa, K., Kubo, K., Kai, M., Hiraga, A., and Watanabe, S. 1999. Effects of splenic red blood cells and blood lactate levels on osmotic fragility of circulating red blood cells in thoroughbred horses during exercise. Jpn. J. Equine Sci. 9: 107-112.

8. Hiro T. 1982. Studies on the osmotic fragility of erythrocytes influenced by a metabolic acidosis. $J$. Physical Fitness Jpn. 31: 279-290.
9. Oyewale,J.O. 1992. Effects of temperature and $\mathrm{pH}$ on osmotic fragility of erythrocytes of the domestic fowl (Gallus domestics) and guinea-fowl (Numida meleagris). Res. Vet. Sci. 52: 1-4.

10. Sakurai, N., Yamaoka, S., and Murakami, M. 1967. Relationship between exercise and changes in blood characteristics in horses. Exp. Rep. Equine Hlth. Lab. 4: 15-19.

11. Shiraki, K. 1968. The effect of splenectomy on sports anemia. J. Physiol. Soc. Jpn. 30: 1-13.

12. Smith, J.E., Erickson, H., and Debowes, R.M. 1989. Changes in circulating equine erythrocytes induced by brief, high-speed exercise. Equine Vet.J. 21: 444446. 